\title{
Peter Hamm, Peter Handke und kein Ende. Stationen einer Annäherung
}

Gauthier Labarthe

\section{(2) OpenEdition}

1 Journals

Édition électronique

URL : http://journals.openedition.org/austriaca/672

DOI : 10.4000/austriaca.672

ISSN : 2729-0603

Éditeur

Presses universitaires de Rouen et du Havre

\section{Édition imprimée}

Date de publication : 1 juin 2018

Pagination : 263-267

ISBN : 979-10-240-1233-9

ISSN : 0396-4590

\section{Référence électronique}

Gauthier Labarthe, "Peter Hamm, Peter Handke und kein Ende. Stationen einer Annäherung ", Austriaca [En ligne], 86 | 2018, mis en ligne le 01 juillet 2020, consulté le 28 janvier 2021. URL : http:// journals.openedition.org/austriaca/672 ; DOI : https://doi.org/10.4000/austriaca.672

Ce document a été généré automatiquement le 28 janvier 2021.

Austriaca. Cahiers universitaires d'information sur l'Autriche 


\title{
Peter Hamm, Peter Handke und kein Ende. Stationen einer Annäherung
}

\author{
Gauthier Labarthe
}

\section{RÉFÉRENCE}

Peter Hamm, Peter Handke und kein Ende. Stationen einer Annäherung, Göttingen, Wallstein, 2017, ISBN : 978-3-8353-4170-8.

1 Julien Gracq écrivait en 1980 dans En lisant, en écrivant que la lecture d'une œuvre littéraire s'apparente à l'« accueil au lecteur de quelqu'un [qui] vous fait les honneurs de son domaine, et de la compagnie duquel il n'est pas question de se libérer ${ }^{1} »$. Peter Hamm nous en livre ici un exemple parfait. Figure de premier plan dans le paysage critique et littéraire en Allemagne, poète, romancier, auteur d'articles, de monographies et de documentaires sur Ingeborg Bachmann, Fernando Pessoa, Heinrich Böll ou bien encore Nelly Sachs pour n'en citer que quelques-uns, il rassemble dans ce livre treize études sur Peter Handke, la plupart publiées sous forme de recensions dans des grands quotidiens (Die Zeit, Focus, Neue Zürchner Zeitung, Der Spiegel), ou bien issues de contributions plus spécialisées. On trouve par exemple un texte paru dans la correspondance entre Hermann Lenz et l'auteur autrichien ( In zweistimmiger Einheit »), ainsi que le discours d'honneur lors de la remise du prix Schiller («Der Geschichtsschreiber der Gegengeschichte oder Die Zurücknahme des Urteils »). Au vu de la diversité des écrits et de la période couverte, des débuts, en 1968, à 2011, il ne faut pas attendre de cet ouvrage une architecture aussi cohérente que le proposerait une monographie par exemple. Il serait d'ailleurs absurde de l'exiger tant le propos et l'enjeu résident ailleurs. Le critique se livre à un exercice qui ne consiste pas tant à réaffirmer sa position dans le champ des études handkéennes, qu'à poser les jalons d'une relation critique, d'un dialogue constant entre un auteur et l'un de ses commentateurs les plus fins. 


\section{La polémique}

2 Les deux premiers articles, publiés dans Die Zeit en 1968, puis dans Konkret en 1969 et qui constituent également les deux premiers textes écrits par Peter Hamm sur Peter Handke, sont à ranger parmi les nombreuses polémiques suscitées par la personnalité de l'auteur autrichien. De ce point de vue, force est de constater qu'il est fait peu de cas de l'œuvre en tant que telle. Passé maître dans l'art de la provocation, Peter Handke ne laisse personne indifférent et Peter Hamm en fait donc ci les frais. Il réagit à un article de l'auteur autrichien dans lequel ce dernier fustigeait la "langue mort-née » (die totgeborene Sprache) du groupe «Culture et Révolution» de l'Association des étudiants socialistes allemands (le SDS, Sozialistischen Deutschen Studentenbunds). Outre le reproche d'utiliser «le jargon du SDS comme prétexte pour faire étalage de son éloquence ${ }^{2}$ », ce qui transparaît ici, notamment à la fin du texte et qui apparaîtra encore plus clairement dans l'article suivant, c'est une critique adornienne adressée à l'encontre des avantgardes littéraires cultivant une image médiatique et prônant une esthétique issue du Pop Art. Déplorant cette « solidarité gâchée » (voir le titre "Versäumte Solidarität ») au profit d'une image médiatique, Peter Hamm explique que l'individualisme forcené caractéristique de « l'intellectuel petit-bourgeois ${ }^{3}$ » révèle l'imposture de cette position marginale affectée par Peter Handke et qui, derrière «les attributs extérieurs de la sous-culture », ne « sert qu'à enrichir la production artistique privée ${ }^{4}$ ».

\section{L'histoire d'une œuvre et de sa critique}

3 Vient ensuite un certain nombre de recensions publiées dans des organes divers. Ces textes centrés sur les œuvres, auxquels on pourrait toutefois reprocher d'être parfois un peu descriptifs, amènent le critique à réviser son jugement. Reconnaissant d'emblée que « pour le public, l'image de [Peter] Handke est bien plus connue que son œuvre » et estimant qu'il "a d'abord été un cas, puis seulement bien plus tard un auteur ${ }^{5}$ », il retrace l'histoire d'un dialogue critique qui perdure aujourd'hui encore. Coïncidant avec une nouvelle phase de l'écriture handkéenne, ces commentaires évoquent la découverte d'une écriture visant à " prôner et à restaurer par la narration un rapport de confiance au monde ", ainsi que le sentiment de la beauté. Passant de la critique à l'adoubement, Peter Hamm attribue à Peter Handke une place de choix dans l'histoire littéraire allemande et mondiale. Il voit en lui le seul auteur qui se soit assigné cette tâche, "la plus difficile et la plus élevée qu'un écrivain ait osé depuis Kafka ${ }^{6}$ », un auteur capable de rédimer ${ }^{7}$, en réconciliant le monde avec les pouvoirs de la littérature, une " époque de l'angoisse, [...] des loups et des ténèbres ${ }^{8}$ ». Les quelques redites qui s'y trouvent, et qui s'expliquent par le projet de cet ouvrage, témoignent du mouvement de la pensée critique dont on voit l'historicité et l'évolution à l'œuvre. Conformément au titre programmatique du livre, Peter Hamm se livre à un jeu d'approche et de distance dont la démarche se rapproche de la paraphrase (Umschreibung) handkéenne qui consiste précisément en un mouvement fait de spirales autour de cette loi énigmatique - cette santes Gesetz empruntée à Stifter - qui creuse le texte sans jamais se laisser saisir. À travers ces variations, le critique fait l'éloge de la patience et de la lenteur, une lenteur qui n'empêche cependant pas les "faux-mouvements » et génère en cela même une autre lisibilité de l'œuvre "que l'on peut feuilleter d'avant en arrière, sans être obligé de la lire chronologiquement ${ }^{9}{ }^{\prime}$. 
Ces tâtonnements de la critique sont magistralement repris dans l'article central, « Der Geschichtsschreiber der Gegengeschichte oder Die Zurücknahme des Urteils », écrit en hommage à Peter Handke pour la remise du prix Schiller. Commentaire le plus important du livre, par sa taille mais surtout par sa profondeur d'analyse, ce texte propose une vision panoramique de l'œuvre handkéenne. Balayant l'ensemble de la production littéraire jusqu'à la plus récente (pour l'époque, en 1994), Peter Hamm nous introduit dans les arcanes de ces textes. Il tisse à partir des motifs de la patience, de la lenteur, du regard, de la métamorphose, de l'événement, de l'épiphanie, de l'enfance et du conteur - pour ne citer que les plus importants - un canevas théorique fin et subtil qui rentre en résonance dans les articles suivants avec de nouveaux interlocuteurs, et non des moindres. Peter Hamm s'efforce d'y reconstruire les liens de filiation qu'entretient Peter Handke avec ses illustres aînés tels que Goethe, Adalbert Stifter, Kafka et plus récemment Hermann Lenz - avec lequel Peter Handke a eu une correspondance fournie publiée en 2006. Et c'est à ce dernier que Peter Handke emprunte le concept de Nebendraußen, si précieux pour comprendre les deux épopées que sont Mon Année dans la baie de personne et La Perte de l'image. Le récit de ces affinités électives place au centre du livre le principe d'amitié que l'auteur autrichien éprouve comme un "amour du monde » qu'il situe au plus profond de lui, mais qu'il ne peut exprimer qu'en se déplaçant dans l'espace vide et hétérotopique de la périphérie ${ }^{10}$.

\section{Histoire d'une non-rencontre}

Le dernier texte délaisse le terrain de la critique pour celui de l'anecdote et de la chronique. En apparence seulement. Peter Hamm y raconte deux rendez-vous avec l'écrivain. Venu deux fois, en 1992, puis en 2002, à chaque fois pour le tournage d'un documentaire, Peter Hamm pénètre dans la maison et découvre l'intérieur de Peter Handke, pourtant absent la seconde fois. L'ouvrage se conclut donc sur cet épilogue qui résume parfaitement l'aventure critique scandée par les non-rencontres, et confrontée en permanence à l'absence, au risque de sombrer dans le monologue. Si le propos détonne par rapport aux autres contributions de l'ouvrage, il est en réalité le plus bel exemple de ce que Peter Hamm discerne dans les épopées, à savoir le «faste du vide». La lecture des deux œuvres épiques s'apparente alors à ce regard de l'étranger qui s'épuise à décrire par le menu les objets et les détails d'un intérieur dans lequel l'hôte est absent et ne se laisse saisir que par ces indices matériels qui creusent et enveloppent le vide. Sous forme de parabole, ce texte éclaire donc la position si ambiguë du critique handkéen, confronté à un auteur qui, tel le Dieu de Simone Weil citée par Peter Hamm, «ne peut être présent dans la création que sous forme d'absence » (p.41), «une absence toute-puissante» (p.47) est-il précisé à un autre moment. Cet ouvrage résonne donc dans les dernières pages comme un vibrant hommage, comme un appel à une étrange communauté d'amis, réunis par l'amour du monde, qui se rendent inaccessibles les uns pour les autres, et qui seraient, en fin de compte, des « amis innés, jurés et jaloux, de [leur] propre solitude profonde de midi et de minuit ${ }^{11} »$. 


\section{NOTES}

1. Julien Gracq, En lisant, en écrivant, Paris, José Corti, 1980, p. 168.

2. «Dass Handke den SDS-Jargon zum Anlass nimmt, um seine Sprachbegabung unter Beweis zu stellen» (p. 10).

3. « [er gibt] sich als der kleinbürgerliche Intellektuelle zu erkennen » (p. 23).

4. "äußerliche Attribute der Subkultur zur Bereicherung der privaten Kunstproduktion zu verwenden $»$ (p. 14).

5. «aber deshalb war beim Publikum Handkes Image immer bekannter als sein Werk. Handke war erst einmal ein Fall, sehr viel später ein Autor » (p. 27).

6. «Peter Handke hat das Schwierigste und Höchste gewagt, was ein Schriftsteller nach Kafka überhaupt wagen konnte, nämlich erzählmend wieder für Weltvertrauen zu werben und Weltvertrauen $z u$ schaffen » (p. 82).

7. Peter Hamm parle en effet de rédemption pour évoquer le lien entre Handke et Kafka : «Weil mir Peter Handkes erzählerisches Werk insgesamt als [...] Erlösung Franz Kafkas, mithin Erlösung des Zeitalters [erscheinen will]» (p. 81).

8. «Zeitalter der Angst, [...] der Wölfe und der Sonnenfinsternis» (p. 79).

9. «ein [...] Buch, in dem man vor- und zurückblättern kann und das man nicht chronologisch lesen muss » (p. 34).

10. Peter Hamm cite Peter Handke lorsqu'il écrit dans Mon Année dans la baie de personne: «Ich habe die Weltliebe. Sie ist in mir. Nur kann ich mir die Weltliebe im Zentrum der Geschichte nichter erhalten. Ich musste dazu an den Rand gehen » (p. 51).

11. Friedrich Nietzsche, Par delà le bien et le mal, dans Euvres complètes, Henri Albert (trad.), Paris, Mercure de France, t. X, p. 83. 